\title{
Alterações hematológicas em pacientes infectados com SARS-CoV-2: uma revisão
}

\section{integrativa}

\author{
Hematologic changes in patients infected with SARS-CoV-2: an integrative review \\ Cambios hematológicos en pacientes infectados con SARS-CoV-2: una revisión integradora
}

Recebido: 22/09/2021 | Revisado: 28/09/2021 | Aceito: 29/09/2021 | Publicado: 01/10/2021

\author{
Amanda de Cássia Castro Santana \\ ORCID: https://orcid.org/0000-0002-5736-7642 \\ Universidade Federal do Pará, Brasil \\ E-mail: amandascastros@gmail.com \\ Chriscia Jamilly Pinto de Sousa \\ ORCID: https://orcid.org/0000-0002-6996-7717 \\ Universidade Federal do Pará, Brasil \\ E-mail: jamillysousa@ufpa.br \\ Carolina Heitmann Mares Azevedo Ribeiro \\ ORCID: https//orcid.org/0000-0002-9457-2733 \\ Universidade Federal do Pará, Brasil \\ E-mail: chribeiro@ufpa.br
}

\begin{abstract}
Resumo
Objetivo: realizar uma síntese integrativa sobre as principais complicações hematológicas correlacionadas à COVID19, com base na análise de artigos e dissertações que abordam a respeito do assunto. Metodologia: foram feitas diversas pesquisas de artigos publicados na íntegra, entre 2020 e 2021, nas bases de dados: Portal de periódicos da Capes; MEDLINE (Medical Literature Analysis and Retrieval System Online); SciELO (Scientific Electronic Library Online) e BVS (Biblioteca Virtual da Saúde); além das diretrizes do Ministério da Saúde (OMS) e os protocolos clínicos e terapêuticos desenvolvidos no Brasil. Após análise dos artigos, foram escolhidos 30 artigos como amostra final para estudo de revisão, além das diretrizes do Ministério da Saúde e outros órgãos mencionados. Resultados: a presente revisão possibilitou observar que, a maior parte dos pacientes que apresentaram casos graves da doença, desenvolveram alterações hematológicas importantes, como a diminuição significativa de plaquetas e lifócitos, aumento do dímero D e prolongamento do tempo de protrombina e tromboplastina. Esses fatores foram ligados a evolução de problemas cardiovasculares como o Tromboembolismo Venoso, Coagulação vascular intradisseminada, muitas vezes, levando o paciente a óbito. Conclusão: esta revisão fornece uma vasta visão da situação atual do COVID-19 durante o último ano e revela, explanando situações interligadas com sua fisiopatologia, manifestações clínicas, as principais características de alterações hematológicas e coagulopáticas observadas no diagnóstico e o impacto da pandemia no sistema de saúde mundial.
\end{abstract}

Palavras-chave: SARS-CoV-2; COVID-19; Alterações hematológicas e coagulopatias.

\begin{abstract}
Objective: to carry out an integrative synthesis of the main hematological complications correlated with COVID-19, based on the analysis of articles and dissertations that address the subject. Methodology: several researches were carried out on articles published in full, between 2020 and 2021, in the following databases: Capes Journal Portal; MEDLINE (Medical Literature Analysis and Retrieval System Online); SciELO (Scientific Electronic Library Online) and VHL (Virtual Health Library); in addition to the guidelines of the Ministry of Health (WHO) and clinical and therapeutic protocols developed in Brazil. After analyzing the articles, 30 articles were chosen as the final sample for the review study, in addition to the guidelines of the Ministry of Health and other mentioned bodies. Results: this review made it possible to observe that most patients who had severe cases of the disease developed important hematological changes, such as a significant decrease in platelets and lymphocytes, increased D-dimer and prolonged prothrombin and thromboplastin time. These factors were linked to the evolution of cardiovascular problems such as Venous Thromboembolism, Intradisseminated vascular coagulation, often leading the patient to death. Conclusion: this review provides a broad overview of the current status of COVID-19 over the past year and reveals, explaining situations interconnected with its pathophysiology, clinical manifestations, the main characteristics of hematological and coagulopathic changes observed in the diagnosis and the impact of the pandemic on the system of world health.
\end{abstract}

Keywords: SARS-CoV-2; COVID-19; Hematological changes and coagulopathies. 


\begin{abstract}
Resumen
Objetivo: realizar una síntesis integradora de las principales complicaciones hematológicas correlacionadas con COVID-19, a partir del análisis de artículos y disertaciones que abordan el tema. Metodología: se realizaron varias investigaciones sobre artículos publicados íntegramente, entre 2020 y 2021, en las siguientes bases de datos: Capes Journal Portal; MEDLINE (Sistema de análisis y recuperación de literatura médica en línea); SciELO (Biblioteca Científica Electrónica en Línea) y BVS (Biblioteca Virtual en Salud); además de las directrices del Ministerio de Salud (OMS) y los protocolos clínicos y terapéuticos desarrollados en Brasil. Resultados: esta revisión permitió observar que la mayoría de los pacientes que presentaban casos graves de la enfermedad desarrollaron importantes cambios hematológicos, como disminución significativa de plaquetas y linfocitos, aumento del dímero D y prolongación del tiempo de protrombina y tromboplastina. Estos factores se vincularon a la evolución de problemas cardiovasculares como el Tromboembolismo venoso, la coagulación vascular intradiseminada, que a menudo conducen al paciente a la muerte. Conclusión: esta revisión ofrece un panorama amplio del estado actual del COVID19 durante el último año y revela, explicando situaciones interconectadas con su fisiopatología, manifestaciones clínicas, las principales características de los cambios hematológicos y coagulopáticos observados en el diagnóstico y el impacto de la pandemia en el sistema de salud mundial.
\end{abstract}

Palabras clave: SARS-CoV-2; COVID-19; Cambios hematológicos y coagulopatías.

\title{
1. Introdução
}

O SARS-CoV-2, responsável pela pandemia global que assolou o mundo no início de 2020 e ainda perdura até os dias atuais, é um vírus da família do Coronavírus, que tem como alvo principal o sistema respiratório humano. A infecção pelo coronavírus (COVID-19) foi identificada pela primeira vez em Wuhan na China em dezembro de 2019 e contribuiu significativamente para uma elevada taxa de mortalidade em vários países, devido ao número de casos de infectados que aumentou exponencialmente em todo o mundo (Zancanaro, 2021).

A sintomatologia da doença do coronavírus na maior parte dos infectados, incluíam sintomas leves semelhantes aos sintomas de uma gripe, como tosse, febre, dor de garganta e mal estar geral. Nesses casos, o próprio sistema imunológico do paciente afetado é responsável pela cura, apenas com tratamentos paliativos, sem necessitar de internação ou medicamentos de alta complexidade. Entretanto, uma menor parte dos afetados apresentou sintomas graves relacionados à doença, tais como: Pneumonia grave, Síndrome do desconforto respiratório agudo (SDRA), choque séptico e falência múltipla de órgãos, o que acarretou em um grave problema de saúde pública mundial.

O SARS-CoV-2 entra na célula através do receptor da enzima conversora de angiotensina 2 presente nos alvéolos e a forma grave de infecção pode ser caracterizada por uma forte resposta imune inflamatória do organismo. Cabe destacar que as plaquetas desempenham um papel central no desenvolvimento de anormalidades da coagulação na sepse, podendo ser ativadas diretamente por mediadores pró-inflamatórios, como o fator ativador de plaquetas, e também podem ser ativadas pela trombina produzida, no qual pode estimular a formação de fibrina por meio de outro mecanismo (Chen et al., 2020).

O dímero-D é um produto da degradação da fibrina e, quando ele aumenta, está associado a uma taxa de mortalidade mais elevada. Com base na análise da experiência clínica e em alguns estudos descritivos, as opiniões dos especialistas enfatizam o papel da hipercoagulabilidade na fisiopatologia do COVID-19, visto que o nível de dímero D aumenta gradualmente à medida que a infecção piora. Um exemplo disso é observado no momento em que a doença evolui para SDRA, n qual é altamente marcada pelos elevados níveis de dímero-D (LI et al., 2020).

Mediante a isso, a referida revisão integrativa objetivou reunir os conhecimentos acerca dos fatores hematológicos relacionados aos casos graves da COVID-19, bem como a evolução de coagulopatias e demais complicações da doença. Portanto, o principal objetivo deste estudo foi realizar uma síntese integrativa sobre as principais complicações hematológicas correlacionadas à COVID-19, com base na análise de artigos e dissertações que abordam a respeito do assunto.

\section{Metodologia}

A revisão integrativa de literatura foi a estratégia utilizada para elaboração desse referido estudo, na qual, suas etapas 
de elaboração foram: definição da pergunta científica; procura de evidências científicas; revisão e seleção dos estudos que serão incluídos no trabalho; análise da qualidade metodológica dos estudos e apresentação dos resultados.

Como estratégia criteriosa de busca e inclusão, foram feitas diversas pesquisas de artigos publicados na íntegra, entre os anos de 2020 e 2021, utilizando os idiomas português, inglês e espanhol.

O processo de busca bibliográfica teve como referência as seguintes bases de dados cientificamente válidas: Portal de periódicos da Capes; MEDLINE (Medical Literature Analysis and Retrieval System Online); SciELO (Scientific Electronic Library Online) e BVS (Biblioteca Virtual da Saúde); além das diretrizes do Ministério da Saúde (OMS) e os protocolos clínicos e terapêuticos desenvolvidos no Brasil. Os descritores utilizados para a coleta de dados da pesquisa foram: SARSCoV-2; COVID-19; alterações hematológicas e coagulopatias

De acordo com o método empregado, foram seguidas as referidas etapas: Elaboração da questão norteadora; amostragem ou busca na literatura; coleta de dados; avaliação dos estudos incluídos; discussão dos resultados e apresentação da revisão (Koche, 2011).

Assim, a principal pergunta do estudo foi: quais os dados disponíveis na literatura científica que explanam acerca dos fatores hematológicos relacionados aos casos graves da COVID-19, bem como a evolução de coagulopatias e outras complicações da doença?

Conforme Figura 1, o procedimento inicial de busca dos artigos para estudo foi realizado pela autora, através das bases de dados mencionadas. Inicialmente, foram excluídos os artigos duplicados e que não estavam disponíveis para acesso na íntegra. Em seguida, procedeu-se à leitura dos artigos, analisando se constavam de informações a respeito da principal pergunta do estudo. Na etapa seguinte, os artigos selecionados foram lidos na íntegra, para avaliar sua seletividade relacionada ao objeto de pesquisa. Os critérios de inclusão definidos para a triagem dos artigos foram: estudos empíricos a respeito das alterações hematológicas em pacientes com casos graves de COVID-19, além de casos que evoluíram para coagulopatias. Foram excluídos artigos que não atendessem a temática desejada.

Figura 1. Fluxograma de identificação e seleção dos artigos para revisão sistemática de literatura sobre alterações nos parâmetros hematológicos na infecção pelo Sars-Cov-2.

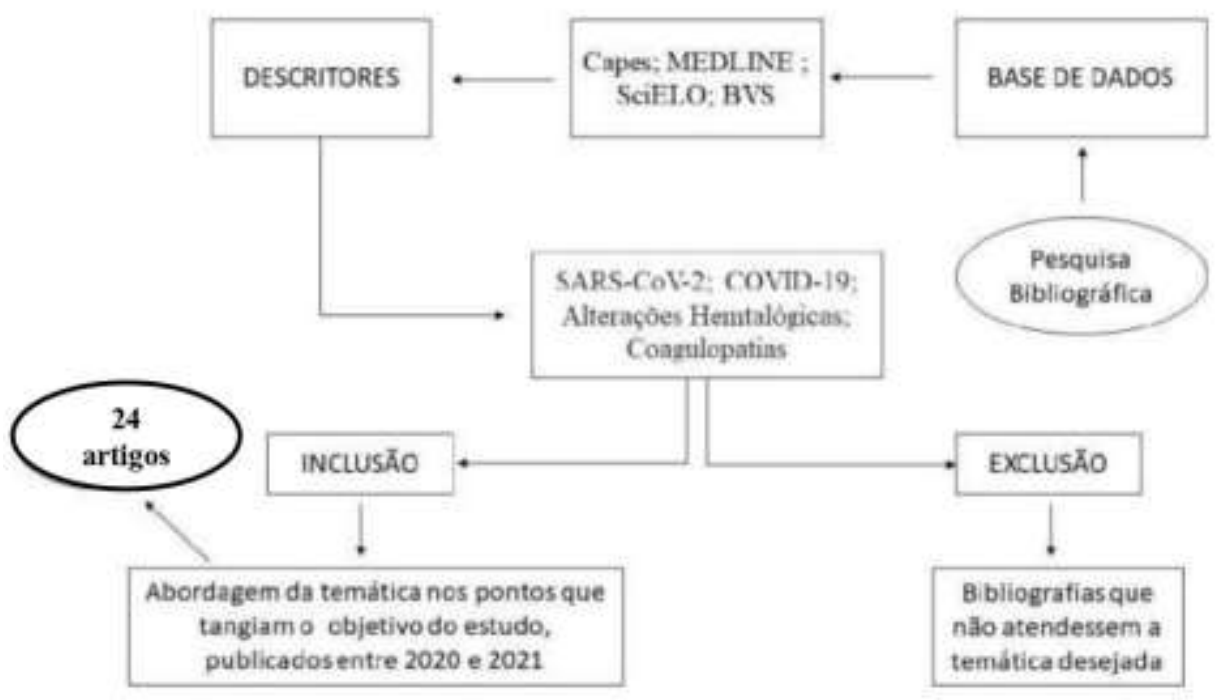

Fonte: Protocolo de Pesquisa, (2021). 


\section{Resultados e Discussão}

De acordo com os descritores utilizados para a pesquisa, encontrou-se na base de dados Portal de periódicos da Capes; MEDLINE (Medical Literature Analysis and Retrieval System Online); SciELO (Scientific Electronic Library Online) e BVS (Biblioteca Virtual da Saúde) em torno de 120 artigos disponíveis em texto completo, na língua portuguesa, inglesa e espanhola. Após a leitura e análise de cada artigo, foram escolhidos cerca de 24 artigos (Tabela 1) como amostra final para estudo de revisão, além das diretrizes do Ministério da Saúde e outros órgãos mencionados, selecionados pelos critérios de inclusão previamente estabelecidos.

Tabela 1. Artigos selecionados para a revisão sistemática de literatura.

\begin{tabular}{|c|c|c|}
\hline $\mathbf{N}^{\mathbf{0}}$ & AUTORES & ARTIGO \\
\hline 1 & Wool, G. D. \& Miller J. L. 2020. & The Impact of COVID-19 Disease on Platelets and Coagulation. \\
\hline 2 & Zancanaro, V. et al., 2021. & $\begin{array}{l}\text { Alterações nos parâmetros hematológicos e imunológicos observadas na infecção pelo sars-cov-2: uma revisão } \\
\text { sistemática de literatura. }\end{array}$ \\
\hline 3 & Prado, M. E. et al., 2021. & $\begin{array}{l}\text { Repercussões hematológicas, cardiovasculares e pulmonares no prognóstico de pacientes infectados por COVID-19: } \\
\text { uma revisão integrativa. }\end{array}$ \\
\hline 4 & Iba, T. et al., 2020. & Coagulopathy in COVID-19. \\
\hline 5 & Paramo, J. A. 2020. & Coagulopatía y trombosis: similitudes y diferencias entre coronavirus patogénicos. \\
\hline 6 & Rossi, F. H. 2020. & Tromboembolismo venoso em pacientes COVID-19. \\
\hline 7 & Arbeláez, G. D et al., 2021. & Complicaciones trombóticas arteriales en pacientes COVID-19. \\
\hline 8 & Mesa, G. E. J. et al., 2021. & Thrombosis and Coagulopathy in COVID-19. \\
\hline 9 & Asakura, H. \& Ogawa, H. 2020. & COVID-19-associated coagulopathy and disseminated intravascular coagulation. \\
\hline 10 & Meaghan, E. C. \& Kanthi, Y. 2020. & COVID-19-associated coagulopathy: An exploration of mechanisms. \\
\hline 11 & Perico, L. et al., 2020. & Immunity, endothelial injury and complement-induced coagulopathy in COVID-19. \\
\hline
\end{tabular}




\begin{tabular}{|c|c|c|}
\hline 12 & Wool, D. G. \& Miller, L. J. 2021. & The Impact of COVID-19 Disease on Platelets and Coagulation. \\
\hline 13 & Dobesh, P. P. \& Trujillo, C. T. (2020). & Coagulopathy, Venous Thromboembolism, and Anticoagulation in Patients with COVID-19. \\
\hline 14 & Mucha, R. S. et al., 2020. & Coagulopathy in COVID-19: Manifestations and management. \\
\hline 15 & Sharif, F. et al., 2021. & $\begin{array}{c}\text { Early hematological indicators of severe COVID-19 disease in hospitalized patients: Data from a South Asian } \\
\text { population. }\end{array}$ \\
\hline 16 & $\begin{array}{l}\text { Rostami, M. \& Mansouritorghabeh, } \\
\text { H. 2020. }\end{array}$ & D-dimer level in COVID-19 infection: a systematic review, \\
\hline 17 & Agbuduwe, C. \& Basu, S. 2020. & Haematological manifestations of COVID-19: From cytopenia to coagulopathy. \\
\hline 18 & Wu, C.; Chen, X. \& Cai, Y. (2020) & $\begin{array}{l}\text { Risk factors associated with acute respiratory dis tress syndrome and death in patients with coronavirus disease } 2019 \\
\text { pneumonia in Wuhan, China. }\end{array}$ \\
\hline 19 & Huang, C.; Wang, Y. \& Li, X. 2020. & Clinical features of patients infected with 2019 novel coronavirus in Wuhan, China. \\
\hline 20 & Zhang, Y. et al., 2020. & Coagulopathy and antiphospholipid antibodies in patients with COVID-19. \\
\hline 21 & Helms, J. et al., 2020. & High risk of thrombosis in patients in severe SARS-CoV-2 infection: a multicenter prospective cohort study. \\
\hline 22 & Tang, N. et al., 2020. & Abnormal coagulation parameters are associated with poor prognosis in patients with novel coronavirus pneumonia. \\
\hline 23 & Nanshan, C. 2020. & $\begin{array}{c}\text { Epidemiological and clinical characteristics of } 99 \text { cases of } 2019 \text { novel coronavirus pneumonia in Wuhan, China: a } \\
\text { descriptive study. }\end{array}$ \\
\hline 24 & Li, K. E., 2020 & The Clinical and Chest CT Features Associated With Severe and Critical COVID-19 Pneumonia. \\
\hline
\end{tabular}

Fonte: Protocolo de Pesquisa, (2021). 
Diante das análises estudadas, a presente revisão possibilitou observar que, o prognóstico dos pacientes que desenvolveram a sintomatologia grave da doença, foi preocupante. Estudos demonstram que a maior parte dos pacientes que apresentaram casos graves da doença, desenvolveram alterações hematológicas importantes, como a diminuição significativa de plaquetas e lifócitos, aumento do dímero D e prolongamento do tempo de protrombina e tromboplastina. Fatores imunológicos também foram observados, como o aumento exacerbado de citocinas pró inflamatórias no soro, o que resultou em uma resposta inflamatória aguda nos pacientes. Esses fatores foram ligados a evolução de problemas cardiovasculares como o Tromboembolismo Venoso, Coagulação vascular intradisseminada, muitas vezes, levando o paciente a óbito. É importante ressaltar, que esses parâmetros foram relacionados a pacientes portadores dos fatores de risco da doença, tais como pacientes obesos, portadores de cardiopatias, pacientes com idade avançada, portadores de doenças cardiorespiratórias, e demais comorbidades.

De acordo com Bikdeli et al., (2020), as alterações hematológicas mais abundantes nos pacientes com COVID-19 grave incluem a trombocitopenia, na qual pode ser classificada como leve (contagem de plaquetas de 100.000 a $150.000 /$ mm3), moderado (50.000 a 99.000/mm3 ) e grave $(<50.000 / \mathrm{mm} 3)$; além dos níveis aumentados de dímero-D. A gravidade da doença é variável e está associada ao prolongamento do tempo de protrombina (TAP), e o tempo de trombina (TT).

É importante salientar que diversos autores citaram em suas pesquisas que, o relato mais frequentemente mencionado está relacionado com os casos de coagulopatias na COVID-19, é justamente uma elevação nos níveis plasmáticos de dímero-d. Diversos estudos têm discutido a respeito da relação entre os níveis elevados de dímero-d e o prognóstico dos pacientes diagnosticados com essas alterações.

Os dímeros-d são produzidos no sangue, sendo resultado da degradação dos polímeros de fibrina estáveis (fibrina reticulada com o fator XIII) pela plasmina. Ou seja, o trombo é formado no corpo pela ativação da coagulação e é decomposto pela ativação da fibrinólise. Diversos estudos evidenciaram que altos níveis de dímero-d estão relacionados com a gravidade. Toda via, se um grande número de coágulos sanguíneos se formar no corpo, mas não se dissolver (representando a condição mais séria do corpo), o aumento do dímero-d pode ser de caráter leve. Dessa forma, é importante explanar que o grau de aumento do dímero-d não está diretamente relacionado à gravidade da condição patológica.

Outros indicadores de coagulopatia no COVID-19 também foram analisados associados como fatores ricos elevados, dentre eles temos: tempo de protrombina, tempo de tromboplastina parcial ativada. Vale ressaltar que os estudos de Klok et al., (2020) não relataram em seus achados níveis elevados de dímero-D no sangue, mas descobriram que os casos de coagulopatia podem ser um fator de risco independente para trombose.

Um estudo multicêntrico de 150 pacientes com COVID-19 na unidade de terapia intensiva em um determinado país na Europa, foi verificado que a taxa positiva de anticoagulantes lúpicos foi muito alta: 50 de 57 pacientes (87,7\%) dos pacientes testados para avaliar o tempo elevado de tromboplastina parcial ativada (Helms et al., 2020). Seguindo sobre essa mesma temática, Tang et al., (2020) evidenciou em seus estudos que a progressão de coagulopatia para coagulação intravascular difusa óbvia (definida pela Sociedade Internacional de Trombose e Hemostasia como pontuação de coagulação intravascular difusa $\geq 5$ pontos) prevê mau prognóstico para os pacientes, ocorrendo em cerca de $71,4 \%$ de todos os não sobreviventes e em $0,6 \%$ nos sobreviventes.

\section{Conclusão}

Diante de todos os resultados das análises bibliográficas, fica evidente que os indicadores hematológicos, incluindo contagem de plaquetas, contagem absoluta de linfócitos, contagem de neutrófilos, NLR e dímero D, desempenham um papel essencial no que se refere ao diagnóstico, estratificação de risco e prognóstico de pacientes hospitalizados por COVID-19 nos estágios iniciais da infecção viral. 
Percebeu-se também que, as alterações no mecanismo de coagulação e formação de fibrina nos níveis pulmonar e sistêmico são achados comuns em todas as infecções por coronavírus, relacionado com a doença pulmonar grave e acompanhado por inflamação sistêmica. Esses fatores corroboram com a alta taxa de complicação no que refere a doenças trombóticas associadas a essas infecções virais, além de possuir um grande impacto no prognóstico dos pacientes, fazendo assim que seja adotado estratégias de prevenção trombótica. Dito isto, sugere-se estudos que realizem o acompanhamento prospectivo de pacientes através da mensuração dos parâmetros citados, a fim de estabelecer um score de prognóstico da doença, que possa vir a subsidiar a tomada de decisão clínica baseada nas melhores evidências.

Dessa forma, de acordo com as análises bibliográficas levantadas durante a produção deste trabalho, é de suma importância relatar que esta revisão integrativa fornece uma vasta visão da situação atual do COVID-19 durante o último ano e revela, explanando situações interligadas com sua fisiopatologia, manifestações clínicas, as principais características de alterações hematológicas e coagulopáticas observadas no diagnóstico e o impacto da pandemia no sistema de saúde mundial.

\section{Referências}

Agbuduwe, C., \& Basu, S. (2020). Haematological manifestations of COVID-19: From cytopenia to coagulopathy. European Journal of Haematology, (15), https://doi.org/10.1111/ejh.13491

Arbeláez, G. D., Vicente, A. M., Sánchez, I. G., Gutiérrez, G. A., Yéboles, C. A., Pérez, C. C., \& Fajardo, G. A. J. (2021). Complicaciones trombóticas arteriales en pacientes COVID-19. Angiología, 73(3), 0003-3170. http://dx.doi.org/10.20960/angiologia.00285.

Asakura, H., \& Ogawa, H. (2020). COVID-19-associated coagulopathy and disseminated intravascular coagulation. Int J Hematol, 1-13. https://dx.doi.org/10.1007\%2Fs12185-020-03029-y

Dobesh, P. P., \& Trujillo, C. T. (2020). Coagulopathy, Venous Thromboembolism, and Anticoagulation in Patients with COVID-19. Pharmacotherapy, 40(11), 1130-1151. https://doi.org/10.1002/phar.2465

Helms, J., Tacquard, D., \& Severac, F. (2020). High risk of thrombosis in patients in severe SARS-CoV-2 infection: a multicenter prospective cohort study. Intensive Care Med, 46(6):1089-1098. 10.1007/s00134-020-06062-x

Huang, C., Wang, Y., \& Li, X. (2020). Clinical features of patients infected with 2019 novel coronavirus in Wuhan, China. Lancet, 395(10223):497-506. $10.1016 / \mathrm{S} 0140-6736(20) 30183-5$

Iba, T., Levy, J. H., Levi, M., \& Thachil, J. (2020). Coagulopathy in COVID-19. J Thromb Haemost, 18: 2103- 2109. https://doi.org/10.1111/jth.14975

Koche, J. C. (2011). Fundamentos de metodologia científica. Petrópolis: Vozes. http://www.brunovivas.com/wpcontent/uploads/sites/10/2018/07/K\%C3\%B6che-Jos\%C3\%A9-Carlos0D0AFundamentos-de-metodologia-cient\%C3\%ADfica-_-teoria-da0D0Aci\%C3\%A Ancia-e-inicia\%C3\%A7\%C3\%A3o-\%C3\%A0-pesquisa.pdfhttps://repositorio.ufsm.br/bitstream/handle/1/15824/Lic_Computacao_Metodologia-PesquisaCientifica.pdf?sequence $=15.4$ )

Klok, F. A., Kruip, M. J., \& Meer, N. J. (2020). Incidence of thrombotic complications in critically ill ICU patients with COVID-19. Thromb Res, 191:145147. 10.1016/j.thromres.2020.04.013

Li, K., Wu, J., Wu, F., Guo, D., Chen, L., Fang, Z., \& Li, C. (2020). The Clinical and Chest CT Features Associated With Severe and Critical COVID-19 Pneumonia. Investigative radiology, 55(6), 327-331. https://doi.org/10.1097/RLI.0000000000000672

Meaghan, E. C., \& Kanthi, Y. (2020). COVID-19-associated coagulopathy: An exploration of mechanisms. Vasc Med, 25(5), 471-478. https://doi.org/10.1177/1358863x20932640

Mesa, G. E. J., Coral, G. S., Montes, C. M., \& Martin, M. J. A. (2021). Thrombosis and Coagulopathy in COVID-19. Curr Probl Cardiol, 46(3), 100742. https://dx.doi.org/10.1016\%2Fj.cpcardiol.2020.100742

Mucha, R. S., Dugar, S., McCrae, K., Joseph, D, Bartholomeww, J., Sacha, L. L. G., \& Militello, M. (2020). Coagulopathy in COVID-19: Manifestations and management. Cleveland Clinic Journal of Medicine August, 87(8), 461-468. https://doi.org/10.3949/ccjm.87a.ccc024

Nanshan, C. (2020). Epidemiologicalandclinicalcharacteristicsof 99 cases of 2019 novel coronavirus pneumonia in Wuhan, China: a descriptivestudy. The Lancet, 395(10223), 507-513.

Paramo, J. A. (2020). Coagulopatía y trombosis: similitudes y diferencias entre coronavirus patogénicos. Anales Sis San Navarra, $43(2)$, $1137-6627$. https://dx.doi.org/10.23938/assn.0885

Perico, L., Benigni, A., Casiraghi, F, Lisa, F. P. N., Renia, L., \& Remuzzi, G. (2020). Immunity, endothelial injury and complement-induced coagulopathy in COVID-19. Nat Rev Nephro, 17(1), 46-64. https://doi.org/10.1038/s41581-020-00357-4

Prado, M. E., Silva, U. F., Júnior, R. G. L. J., Rocha, Q. M. L., Petrola, S. N. L., Pereira, C. M. B., Costa, A. D., \& Hall, R. P. (2021). Repercussões hematológicas, cardiovasculares e pulmonares no prognóstico de pacientes infectados por COVID-19: uma revisão integrativa/ Hematological, cardiovascular and pulmonary repercussions in the prognosis of patients infected by COVID-19: a literary review. Brazilian Journal of Health Review, Vol 4 (1): $2525-8761$. https://doi.org/10.34119/bjhrv4n1-137 
Research, Society and Development, v. 10, n. 12, e578101220932, 2021

(CC BY 4.0) | ISSN 2525-3409 | DOI: http://dx.doi.org/10.33448/rsd-v10i12.20932

Rossi, F. H. (2020). Tromboembolismo venoso em pacientes COVID-19. J. Vasc. Bras, 2020,19: e20200107. https://doi.org/10.1590/1677-5449.200107

Rostami, M., \& Mansouritorghabeh, H. (2020) D-dimer level in COVID-19 infection: a systematic review, Expert Review of Hematology, 13:11, 1265-1275, https://doi.org/10.1080/17474086.2020.1831383

Sharif, F., Khan, S., Junaid, A., Jahangir, S., Saeed, M., Ijaz, M., Ahmad, N. I., \& Kamran, S. (2021). Early hematological indicators of severe COVID-19 disease in hospitalized patients: Data from a South Asian population. Int J Lab Hematol, 10.1111/ijlh.13533. https://dx.doi.org/10.1111\%2Fijlh.13533

Tang, N., Li, D., Wang, X., \& Sun, Z. (2020). Abnormal coagulation parameters are associated with poor prognosis in patients with novel coronavirus pneumonia. J Thromb Haemost, 18(4):844-847. 10.1111/jth.14768

Wool, G. D., \& Miller J. L. (2020). The Impact of COVID-19 Disease on Platelets and Coagulation. Pathobiology. 13, 1-13. https://dx.doi.org/10.1159\%2F000512007

Wool, D. G., \& Miller, L. J. (2021). The Impact of COVID-19 Disease on Platelets and Coagulation. Pathobiology, 88(1):15-27. https://doi.org/10.1159/000512007

Wu, C., Chen, X., \& Cai, Y. (2020). Risk factors associated with acute respiratory dis tress syndrome and death in patients with coronavirus disease 2019 pneumonia in Wuhan, China. JAMA Intern Med, 200994. 10.1001/jamainternmed.2020.0994

Zancanaro, V., Moura, R. T., Bellaver, H. E,. \& Javorski, M. J. (2021). Alterações nos parâmetros hematológicos e imunológicos observadas na infecção pelo sars-cov-2: uma revisão sistemática de literatura. Brazilian Journal of Development, 7 (5): 2525-8761. https://doi.org/10.34117/bjdv.v7i5.30097

Zhang, Y., Xiao, M., \& Zhang, S. (2020). Coagulopathy and antiphospholipid antibodies in patients with COVID-19. N Engl J Med, 382(17):e38. 10.1056/NEJMc2007575 